\title{
Analysis of Bioproduct Separation Using Gel-Enclosed Adsorbents
}

\begin{abstract}
The concept of "gel-enclosed" adsorbents was recently introduced for whole fermentation broth processing. The term "gel-enclosed adsorbents" in this paper refers to small adsorbent particles such as the ones used in affinity or ion-exchange chromatography enclosed within a protective, spherical, semipermeable gel barrier. The barrier can be in the form of an entrapment matrix where a porous gel matrix in itself contains entrapped adsorbent particles, or in the form of an encapsulating membrane where an aqueous spherical core containing the adsorbent particles is enclosed by a gel membrane. Both experimental and theoretical analyses were carried out to elicit a more fundamental view of the diffusion and adsorption phenomena occurring within these forms of adsorbents. Furthermore, the effective porosity of the gel matrix and the effective diffusivities of the species being separated, as well as their adsorption behavior combined result in what is here termed "kinetic selectivity."
\end{abstract}

\author{
Somesh C. Nigam \\ Ali R. Siahpush \\ Henry Y. Wang \\ Department of Chemical Engineering \\ University of Michigan \\ Ann Arbor, Ml 48109
}

\section{Introduction}

The use of gel-enclosed adsorbents for bioproduct recovery from whole broths has recently been introduced (Wang and Sobnosky, 1986; Nigam et al., 1988). The two different system morphologies are that of gel-enclosed adsorbent beads which consist of small adsorbent particles (such as those used in chromatography) entrapped within a porous spherical gel matrix (Figure 1a), and gel-enclosed adsorbent capsules which consist of adsorbent particles encapsulated within a thin (50$100 \mu \mathrm{m})$ spherical gel membrane. The pore size of the gel can be tailored to the size of the desired protein product in the broth, excluding many undesired products from contacting the adsorbent. This same protective gel prevents adsorbent contact with suspended solids and colloidal contaminants, adding a great degree of control to a traditional separation process.

The binding of bioproducts onto gel-enclosed adsorbents depends on a number of design parameters. The performance of a gel-enclosed adsorbent preparation in a dynamic biochemical separation process can be evaluated by judging the adsorption rate and the selectivity for the target bioproduct (Nigam et al., 1988). The dynamic adsorption behavior of different species is controlled by various diffusional resistances in addition to the intrinsic binding characteristics of the free adsorbent particles.

Correspondence concerning this paper should be addressed to $H$. $Y$. Wang.
Diffusional characteristics in turn are influenced by design parameters such as size of the bead or capsule, capsule membrane thickness, adsorbent content of the bead or capsule and the type of hydrogel used. In the area of biochemical separations the classical adsorption processes rely mainly on the differences in equilibrium binding of various components.

It is the purpose of this study to demonstrate the feasibility of utilizing gel-enclosed adsorbents to enhance the binding of a small target molecule in a crude solution containing an excess of larger molecular weight adsorbable contaminants and to develop a simplified model for the two component case, where the target protein exists within the broth in the presence of an excess amount of only one contaminant. As in the recovery of many recombinant proteins, the target protein's concentration is expected to be in the $\mu \mathrm{g} / \mathrm{mL}$ range, while the contaminants are expected to be in the $\mathrm{mg} / \mathrm{mL}$ concentration range. It is not expected that such a simple model would be used to accurately predict the batch concentration profiles in a complex multicomponent mixture. The mathematical analysis of bioproduct adsorption from a complex multicomponent mixture using immobilized adsorbents is significantly more difficult because the bioproduct has to compete for binding sites with all of the adsorbable contaminants having different diffusional and binding properties. However, the problem can be reduced down to a two-component diffusion and binding problem, and can be mathematically analyzed using simplifying assumptions if the 
Gel Membrane

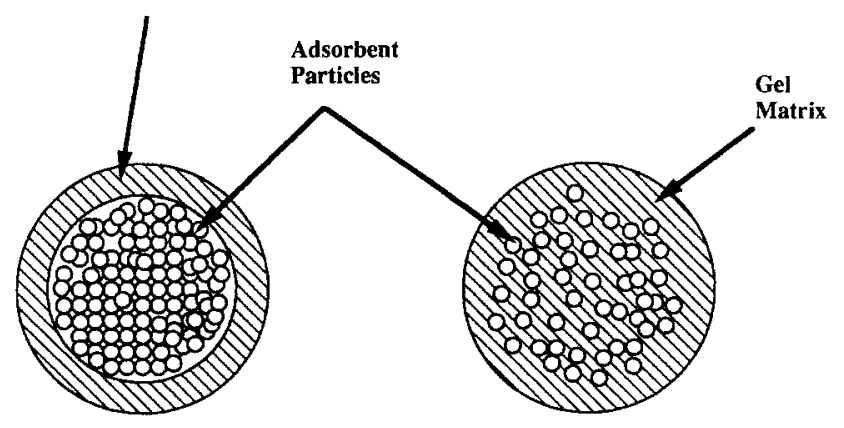

Figure 1a. Gel-enclosed adsorbents.
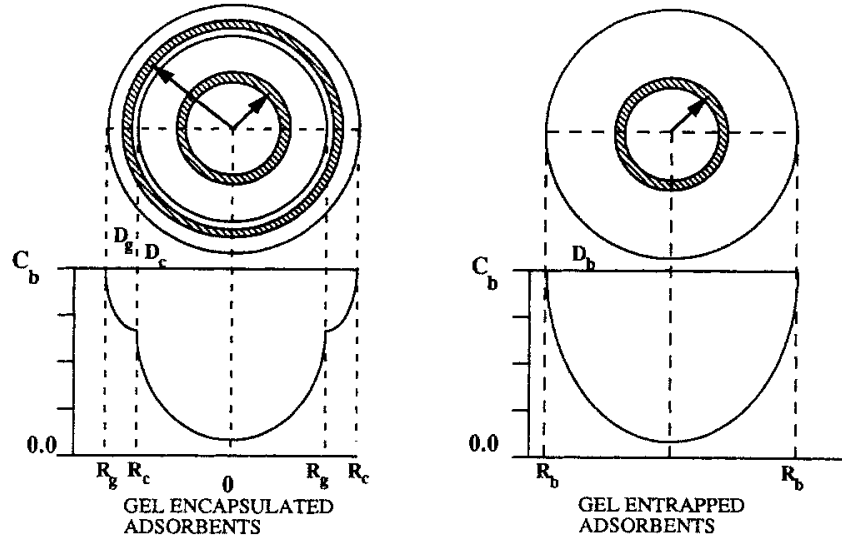

Figure 1b. Profile of mass transfer in gel-enclosed adsorbents.

binding isotherms and diffusional coefficients of the two competing species are well characterized. Such a model may also serve as a useful tool to arrive at an initial set of design parameters even in relatively complex separations.

A greater degree of separation is possible by using gelenclosed adsorbents than by using free adsorbents because of the differences in the rates of diffusion and adsorption. The small target molecule is able to diffuse and adsorb at a faster rate compared to larger molecular weight contaminants. As the incubation time increases, however, the target molecule is desorbed out while slower diffusing contaminants progressively saturate the binding sites. It is evident that an optimum separation time exists in such a way that longer contact of these adsorbents will actually result in a reduction of the total amount of target protein removed from the broth. This gives rise to the concept of "kinetic selectivity." Our previous experimental data using gel-enclosed adsorbents demonstrate this concept with $\beta$-Lactamase as the model bioproduct (Nigam et al., 1988). In this paper the term "kinetic-selectivity" refers to the timedependent ratio of the adsorbed concentration of target protein to the total adsorbed contaminant concentration and is described mathematically as follows:

$$
\text { Kinetic Selectivity }=\frac{C_{s p}(t)}{\sum_{i=1}^{n} C_{s i}(t)}
$$

In principle, the kinetic selectivity for a particular bioproduct can be optimized by manipulating the different diffusional resistances and adsorption time (Nigam et al., 1988).

\section{Model}

Figure 1 illustrates the volumetric elements used to formulate material balance equations for spherical gel-matrix-entrapped and gel-membrane-encapsulated adsorbents used in this study. Several assumptions were utilized to develop this model. Adsorbent particles were assumed to be distributed uniformly within the core of the capsules and gel beads. The geometry of gel-membrane-encapsulated and gel-matrix-entrapped adsorbents was spherical. The external mass transfer due to boundary layer was assumed to be negligible if the bulk solution were well stirred. This assumption is supported by the experimental observations of Tanaka et al. (1984). Furthermore, diffusion of all of the species is considered to follow Fickian laws, and the rate of adsorption is assumed to be much faster than the diffusion rate, causing local adsorption equilibrium. Such an assumption will no longer hold if the bead or capsule size is reduced to a point where the diffusion and adsorption time constants are of the same order of magnitude. The size of beads and capsules in this model system is in the range of approximately $1 \mathrm{~mm}$, while the adsorbent particle size is in the range of 50-70 $\mu \mathrm{m}$. In this range, the adsorption and diffusion time constants are at least two orders of magnitude different.

\section{Gel-matrix-entrapped adsorbents}

For theoretical analysis, a single spherical bead is considered here. Each component is assumed to have a constant effective diffusivity throughout the bead. For the sake of simplicity, the effective diffusivity is used as a lumped parameter for the entire matrix of the gel-entrapped adsorbent bead. Equations 2 to 8 describe the material balances for gel-entrapped beads. These equations can be written in dimensionless form as follows:

Dynamic Mass-Balances within the Bead:

$$
\frac{\epsilon_{b i}}{\bar{R}^{2}} \frac{\partial}{\partial \bar{R}}\left(\bar{R}^{2} \frac{\partial \bar{C}_{i}}{\partial \bar{R}}\right)=\epsilon_{b i}\left(\frac{D_{b i}}{D_{b i}}\right) \frac{\partial \bar{C}_{i}}{\partial \bar{t}}+\gamma_{b i}\left(\frac{D_{b 1}}{D_{b i}}\right) \frac{\partial \bar{C}_{s i}}{\partial \bar{t}}
$$

where

$$
\gamma_{b i}=\left(\frac{\rho_{b} C_{s i m}}{C_{i m}}\right)
$$

Equilibrium binding isotherms for free adsorbents relating the adsorbed concentration of $i$ th component as a function of the concentrations of $n$ competing species:

$$
\bar{C}_{s i}=f\left(\bar{C}_{1}, \bar{C}_{2}, \ldots, \bar{C}_{n} ; C_{1 m}, C_{2 m}, \ldots, C_{n m}\right)
$$

Bulk Concentration in the Batch Adsorption System:

$$
\frac{\partial \bar{C}_{b i}}{\partial \bar{t}}=-\frac{3}{\alpha}\left(\frac{D_{i}}{D_{1}}\right)\left[\frac{\partial \bar{C}_{i}}{\partial \bar{R}}\right]_{\bar{R}=1}
$$

where

$$
\alpha=\frac{V}{\frac{4}{3} \pi R_{b}^{3} N}
$$


Boundary Conditions:

$$
\begin{aligned}
& {\left[\frac{\partial \bar{C}_{i}}{\partial \bar{R}}\right]_{\bar{R}=0}=0} \\
& {\left[\bar{C}_{i}\right]_{\bar{R}=1}=\bar{C}_{b i}}
\end{aligned}
$$

Initial Conditions:

$$
\begin{gathered}
{\left[\bar{C}_{i}\right]_{0 \leq \bar{R}<1}=0} \\
\bar{C}_{b i}=1
\end{gathered}
$$

Dimensionless Variables:

$$
\begin{aligned}
\bar{C}_{i}=\frac{C_{i}}{C_{i m}} ; \quad \bar{C}_{s i}=\frac{C_{s i}}{C_{s i m}} ; \quad \bar{C}_{b i}= & \frac{C_{b i}}{C_{i m}} ; \\
& \bar{R}=\frac{R}{R_{b}} ; \quad \bar{t}=\frac{t}{t^{*}} ; \quad t^{*}=\frac{R_{b}^{2}}{D_{b 1}}
\end{aligned}
$$

An examination of the dimensionless equations reveals the significance of several dimensionless parameters in determining the relationship between dimensionless bulk concentration and the dimensionless time for different adsorbing components. These parameters include the ratios of the effective product diffusivity to that of each contaminant, porosity and the dimensionless adsorption capacity of the bead for each component. The concentration profile also depends on $\alpha$, which is the ratio of the liquid volume to the total bead volume in the batch.

\section{Gel membrane encapsulated adsorbents}

The formulation of the mahematical model for gel-membraneencapsulated adsorbents differs from that for gel-entrapped adsorbents only by an extra set of dynamic mass-balance equations describing the transport of various species in the enclosing gel membrane. Each component is assumed to have a constant, but different, diffusivity throughout the capsule core and the membrane. Equations 9-17 represent the material balances in gel-membrane-encapsulated adsorbents in the nondimensional form.

\section{Dynamic Mass Balances within the Capsule Core:}

$$
\frac{\epsilon_{c i}}{\bar{R}^{2}} \frac{\partial}{\partial \bar{R}}\left(\bar{R}^{2} \frac{\partial \bar{C}_{i}}{\partial \bar{R}}\right)=\epsilon_{c i}\left(\frac{D_{c i}}{D_{c i}}\right) \frac{\partial \bar{C}_{i}}{\partial \bar{t}}+\gamma_{c i}\left(\frac{D_{c 1}}{D_{c i}}\right) \frac{\partial \bar{C}_{s i}}{\partial \bar{t}}
$$

where

$$
\gamma_{c i}=\left(\frac{\rho_{c} C_{s i m}}{C_{i m}}\right)
$$

Dynamic Mass Balance in the Gel Membrane:

$$
\frac{1}{\bar{R}^{2}} \frac{\partial}{\partial \bar{R}}\left(\bar{R}^{2} \frac{\partial \bar{C}_{i}}{\partial \bar{R}}\right)=\epsilon_{g i}\left(\frac{D_{c 1}}{D_{g i}}\right) \frac{\partial \bar{C}_{i}}{\partial \bar{t}}
$$

Equilibrium binding isotherms for free adsorbents relating the adsorbed concentration of the $i$ th component as a function of the concentrations of $n$ competing species:

$$
\bar{C}_{s i}=f\left(\bar{C}_{1}, \bar{C}_{2}, \ldots, \bar{C}_{n} ; C_{1 m}, C_{2 m}, \ldots, C_{n m}\right)
$$

Bulk Concentration in the Batch Adsorption System:

$$
\frac{\partial \bar{C}_{b i}}{\partial \bar{t}}=-\frac{3}{\alpha}\left(\frac{R_{c}}{R_{g}}\right)\left(\frac{D_{g i}}{D_{c 1}}\right)\left[\frac{\partial \bar{C}_{i}}{\partial \bar{R}}\right]_{\bar{R}=\frac{R_{g}}{R_{c}}}
$$

where

$$
\alpha=\frac{V}{\frac{4}{3} \pi R_{g}^{3} N}
$$

Note that all diffusivities listed are effective diffusion coefficients that lump diffusion in the matrix as well as diffusion within the adsorbent particles.

\section{Boundary Conditions:}

$$
\begin{gathered}
{\left[\frac{\partial \bar{C}_{i}}{\partial \bar{R}}\right]_{\bar{R}=0}=0} \\
-\left[\frac{\partial \bar{C}_{i}}{\partial \bar{R}}\right]_{\bar{R}=1^{+}}=-\left(\frac{D_{g i} / D_{c 1}}{D_{c i} / D_{c 1}}\right)\left[\frac{\partial \bar{C}_{i}}{\partial \bar{R}}\right]_{\bar{R}=1-} \\
{\left[\bar{C}_{i}\right]_{\bar{R}=R_{g} / R_{c}}=\bar{C}_{b i}}
\end{gathered}
$$

Initial Conditions:

$$
\begin{gathered}
{\left[\bar{C}_{i}\right]_{0 \leq \bar{R}<R_{g} / R_{c}}=0} \\
\bar{C}_{b i}=1
\end{gathered}
$$

Dimensionless Variables:

These remain unchanged, with the exception of the following:

$$
\bar{R}=\frac{R}{R_{c}} ; \quad t^{*}=\frac{R_{c}^{2}}{D_{c 1}}
$$

The relationship of dimensionless bulk concentration with respect to dimensionless time in this case depends on a greater number of dimensionless parameters compared to those in the case of gel-entrapped adsorbent beads. Several parameters such as ratios of the diffusion coefficient of the product to that of each contaminant and porosity for each component need to be determined in the capsule core and the gel membrane.

The above equations in dimensionless form can now be solved using the finite-difference method (Carnahan et al., 1969) to obtain the bulk concentration profiles of various adsorbing species. To apply this mathematical model, we need to estimate the equilibrium adsorption characteristics, diffusion coefficients of each competing component in different regions, bead/capsule design parameters such as adsorbent content, porosity and geometric characteristics including size, shape, and membrane thickness. These were obtained experimentally and were systemspecific in this investigation. The two-component model system 
chosen consists of gel-entrapped and membrane-encapsulated DEAE-Trisacryl using solutions containing two proteins: $\beta$-lactamase, human serum albumin, and their mixtures (Nigam, 1988). The manufacture of various types of immobilized adsorbents, their utilities and other experimental details have been described elsewhere (Nigam, 1988; Nigam et al., 1988). The model discussed above can also be utilized to analyze complex multicomponent systems. However, the use of the mathematical model developed in this paper in analyzing highly complex multicomponent solutions is limited by the difficulties in experimentally determining adsorption isotherms and diffusivities for the bioproduct and each significant contaminant.

\section{Determining Parameter Values in Protein Adsorption Using Gel-Enclosed Adsorbents}

\section{Characterization of equilibrium adsorption}

Simple equilibrium binding models such as Langmuir or Freundlich isotherms often fail to provide an adequate description of complex binding interactions. This is especially true in the case of protein adsorption on ion-exchange-type adsorbents where the binding may depend strongly on the buffer conditions and involves multipoint attachment to the adsorbent surface. Thus, for a most reliable description, equilibrium adsorption relationships need to be determined experimentally for any given set of physico-chemical conditions. In the binary experimental system chosen albumin is present in excess $(10 \mathrm{mg} / \mathrm{mL})$ over the model bioproduct, $\beta$-lactamase $(0.32 \mu \mathrm{g} / \mathrm{mL})$. Since the concentration of albumin is over four orders of magnitude higher than that of $\beta$-lactamase, there is a negligible effect of $\beta$-lactamase concentration on the adsorption isotherm of albumin. However, in this concentration range the adsorption isotherm of $\beta$-lactamase depends strongly on the concentration of albumin.

$\beta$-Lactamase Adsorption on DEAE-Trisacryl. Figure 2 shows the equilibrium adsorption isotherm of purified $\beta$-lactamase on DEAE-Trisacryl adsorbents. In absence of albumin, $\beta$-lactamase adsorption isotherm was found to be almost linear in the

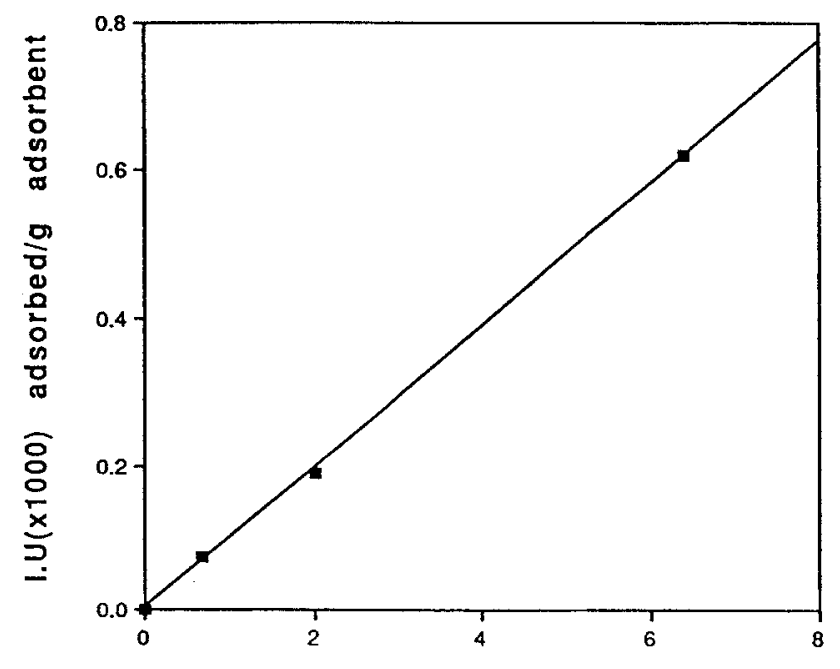

Equilibrium Conc. $1 . \mathrm{U} . / \mathrm{ml}$

Figure 2. Equilibrium adsorption isotherm of $\beta$-lactamase on DEAE-trisacryl.

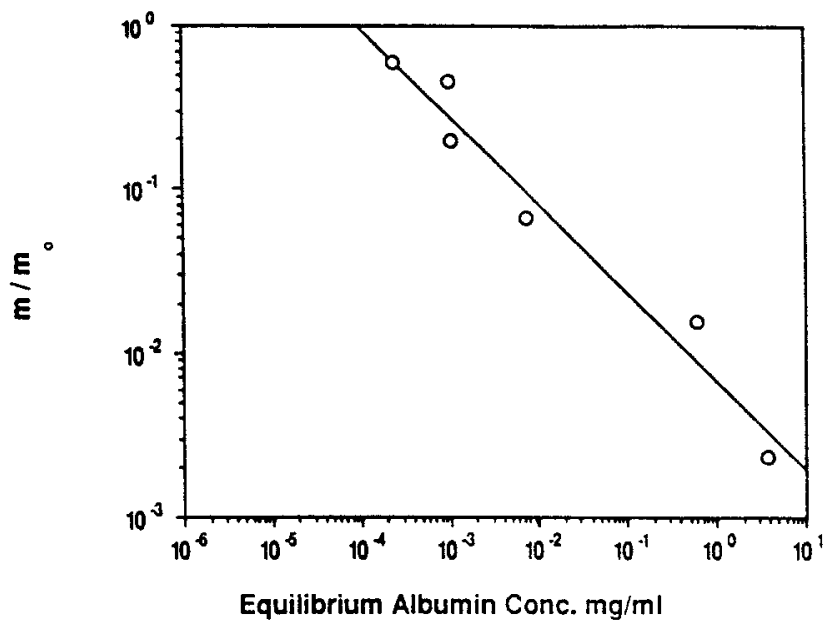

Figure 3. Effect of albumin concentration on the slope of $\beta$-lactamase adsorption isotherm.

concentration range of interest $(<3$ I.U./mL or approx. 0.5 $\mu \mathrm{g} / \mathrm{mL}$ ). For the sake of simplicity it was assumed that the adsorption isotherm of $\beta$-lactamase remains linear even in the presence of albumin. Consequently, the addition of albumin would influence the $\beta$-lactamase adsorption isotherm by decreasing its slope. To characterize this effect, batch equilibrium experiments were carried out with a known quantity of DEAETrisacryl adsorbent which was exposed to a fixed liquid volume containing the same initial concentration of $\beta$-lactamase $(1.94$ I.U. $/ \mathrm{mL} ; 0.32 \mu \mathrm{g} / \mathrm{mL}$ ) and increasing concentrations of albu$\min (0$ to $10 \mathrm{mg} / \mathrm{mL})$. As the concentration of albumin in the solution increased, the ratio of the adsorbed to free equilibrium concentration of $\beta$-lactamase, $m$, declined due to increased competition. Figure 3 shows the dependence of the slope on albumin concentration in the bulk. Table 1 shows the form the mathematical function used to empirically fit this data.

Albumin Adsorption on DEAE-Trisacryl. In the experimental concentration range, the equilibrium adsorption isotherm of albumin was found to be independent of the $\beta$-lactamase concentration. Figure 4 shows the experimentally obtained albumin adsorption isotherm. The experimental data can be satisfactorily correlated using bilinear Freundlich-type isotherm. Table 2 shows the parameters for the two Freundlichtype isotherms employed to correlate albumin adsorption data. The concentration of the adsorbed albumin increased to about $50 \mathrm{mg} / \mathrm{g}$ adsorbent as the concentration in the free solution increased to about $3 \mathrm{mg} / \mathrm{mL}$. This type of highly nonlinear equilibrium adsorption behavior is similar to that obtained for the adsorption of bovine serum albumin on cellulosic ion exchangers in the studies conducted by Graham and Fook (1982).

\section{Characterization of bead/capsule design parameters}

Table 3 shows the values of the bead/capsule design parameters required to simulate the adsorption behavior of the different types of gel-enclosed adsorbent preparations employed in this study.

Geometric Dimensions. The geometric dimensions were measured by a microscope equipped with a micrometer scale. The bead and capsule sizes were estimated by measuring the volume of buffer displaced by a known number of beads in a volumetric 
Table 1. Mathematical Relationship: Slope of $\boldsymbol{\beta}$-Lactamase Adsorption Isotherm vs. Albumin Concentration

$$
\begin{aligned}
& C_{s 1}=m C_{1} \\
& \frac{m}{m_{o}}=1.0 ; \quad C_{2}<9.6 \times 10^{-5} \mathrm{mg} / \mathrm{mL} \\
& \frac{m}{m_{o}}=6.82 \times 10^{-3} C_{2}^{-0.539} ; \quad C_{2}>9.6 \times 10^{-5} \mathrm{mg} / \mathrm{mL} \\
& m_{o}=94.43 \mathrm{~g} \text { adsorbent } / \mathrm{mL}
\end{aligned}
$$

flask. The manufacturing method for these beads was optimized in our laboratory to result in a nearly monodisperse population $(1.2-1.4 \mathrm{~mm})$; without this, the volumetric measurement of diameter would merely result in a population average.

Adsorbent Content in the Capsules or Beads. Adsorbent content $\left(\rho_{b}\right)$ in the agarose beads was determined by recovering the adsorbent particles from a known amount of gel-entrapped adsorbent beads after liquifying the agarose matrix by increasing temperature to approximately $45^{\circ} \mathrm{C}$. The adsorbent content of the alginate capsules $\left(\rho_{c}\right)$ was determined similarly by using EDTA to dissolve the capsule membrane. Recovered adsorbent particles were extensively washed with distilled water to remove the contaminants. The adsorbent content of the capsules or beads was determined by measuring the dry weight or moist weight of the recovered adsorbent particles.

Porosity of the Beads or Capsules in Different Regions. The porosity of the bead or capsule differs for different adsorbing components. Porosity in this model is defined as the effective free volume seen by a particular component within a porous matrix. Porosity within alginate membrane was found using independent experiments involving the equilibrium dilution of $\beta$-lactamase and albumin into a known volume of $\mathrm{Ca}$-alginate gel beads (without adsorbent particles) in batch mode. Porosity of $\beta$-lactamase in the DEAE-Trisacryl/agarose beads and the core of DEAE-Trisacryl/alginate capsules were estimated by measuring the equilibrium dilution of $\beta$-lactamase using a known volume of beads or capsules from a finite batch containing a high concentration of albumin $(10 \mathrm{mg} / \mathrm{mL})$. The high concentration of albumin compared to $\beta$-lactamase ensured that the adsorption of $\beta$-lactamase was negligible and that $\beta$-lactamase dilution

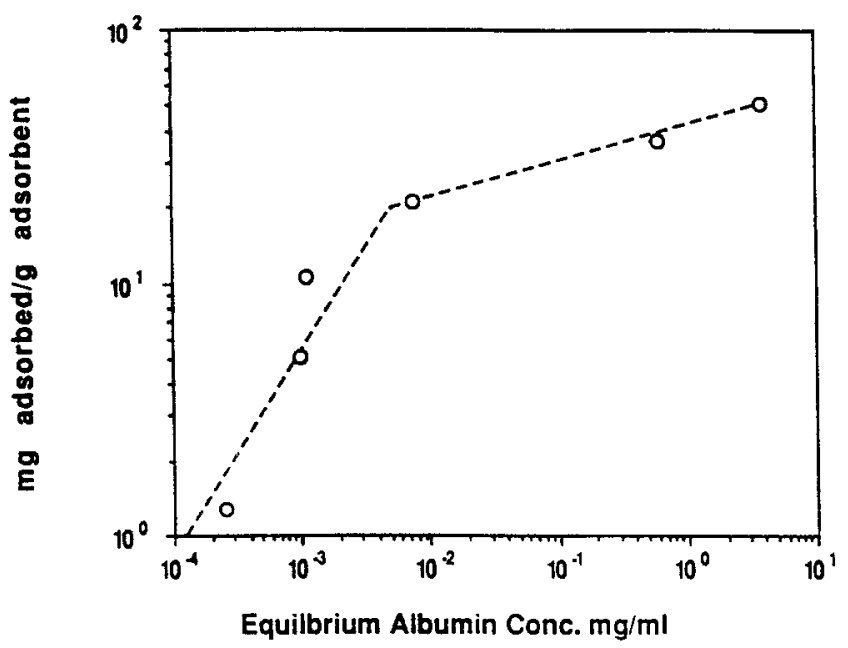

Flgure 4. Equilibrium adsorption isotherm of albumin on DEAE-trisacryl.
Table 2. Mathematical Relationships Describing Albumin Adsorption Isotherm

$C_{s 2}=1329.3 C_{2}^{0.8} ; \quad C_{2}<5.14 \times 10^{-3} \mathrm{mg} / \mathrm{mL}$

$C_{s 2}=42.2 C_{2}^{0.1455} ; \quad C_{2}>5.14 \times 10^{-3} \mathrm{mg} / \mathrm{mL}$

was due primarily to the pore volume in the beads or capsules. Similarly, the porosity of albumin in DEAE-Trisacryl/agarose beads and the core of DEAE-Trisacryl/alginate capsules were estimated by measuring the equilibrium dilution of a highconcentration albumin solution in a finite batch containing a known volume of presaturated immobilized adsorbents. Greater care had to be taken to ensure the attainment of equilibrium for albumin which is the slower diffusing component. More accurate measurement and determination of these porosity terms remain to be explored.

\section{Characterization of diffusional properties}

The successful application of this model requires an estimate of the diffusivities of different competing components in the different regions of immobilized adsorbents. It is difficult to independently estimate effective diffusivities in the regions containing closely packed adsorbent particles such as within the agarose bead and the core of the alginate capsules because the mass transport is always influenced by adsorption effects. This problem can be bypassed by utilizing diffusion coefficient as the only adjustable parameter in the mathematical model to fit the experimentally generated kinetic bulk concentration profiles. This approach has been utilized by a number of investigators in studying adsorption in a variety of porous adsorbents (Tsou and Graham, 1985; Graham and Fook, 1982; Horstmann and Chase, 1989).

In the case of alginate capsules, the diffusivities of $\beta$-lactamase and albumin within the alginate membrane are also required. These values were estimated using independent batch kinetic experiments involving the diffusion of $\beta$-lactamase or albumin into calcium alginate gel beads without any adsorbent particles using the methodology described by Tanaka (1984).

\section{Experimental Results vs. Computer Simulations}

An artificial crude solution comprising $0.31 \mu \mathrm{g} / \mathrm{mL} \beta$-lactamase (model bioproduct) and $10 \mathrm{mg} / \mathrm{mL}$ human serum albumin (model contaminant) in 25mM HEPES was utilized for investi-

Table 3. Bead/Capsule Design Parameters Used to Simulate Adsorption Experiments

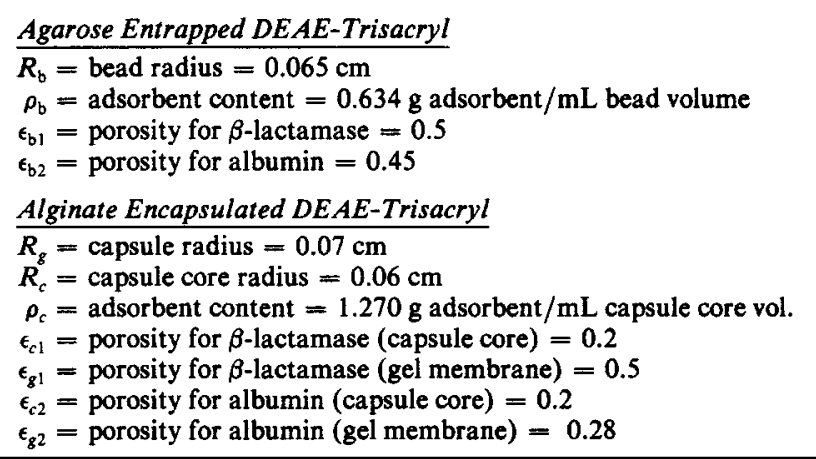


gating two-component competitive diffusion and adsorption. Concentration of albumin was deliberately chosen to be in excess of $\beta$-lactamase (approximately 4 orders of magnitude higher). The numerical simulation of adsorption using gelencapsulated adsorbents presents greater complexity because an additional set of partial differential equations is needed to describe mass transport in the gel membrane. Moreover, in this situation we require diffusivity values for the adsorbing component in both the gel membrane and the capsule core. The diffusivities of both $\beta$-lactamase and albumin in the different regions of the immobilized adsorbent preparations were either estimated independently or utilized as the only adjustable parameter. A parameter sensitivity analysis was carried out to ensure that the model solutions were stable to changes in the adjusted diffusion coefficients. By stable, it is meant that a $100 \%$ change in the adjustable parameter resulted in a change $(\leq 100 \%)$ in the magnitude of the solution. The magnitude of the largest deviation in the solution produced as a result of an $800 \%$ change in the adjustable parameter was $25 \%$, this ensures that reliable values can be calculated for the diffusivities as described. Figures 5 and 6 show the sensitivity of the bulk concentration of $\beta$-lactamase on the adjustable diffusivities. The solution sensitivity in the case of albumin was comparable to that of $\beta$-lactamase.

Figure 7 shows a comparison between experimental and simulated concentration profiles for the competitive diffusion and adsorption of $\beta$-lactamase and albumin in agarose entrapped DEAE-Trisacryl adsorbents while Figure 8 shows the same comparison for diffusion and adsorption in alginateencapsulated DEAE-Trisacryl. As a measure of comparison between experimental error and model sensitivity, the experimental error in all data presented was approximately $\pm 2.5 \%$, which in graphical form would produce error bars of the same magnitude as the symbols used. The corresponding diffusion coefficients used in the model appear in Table 4. Simulation for the encapsulated adsorbents requires diffusivities in both the membrane and the capsule core for all diffusing components. The diffusion coefficients within the membrane were estimated from independent batch kinetic experiments involving the diffusion of substrates from the model crude solution into spherical

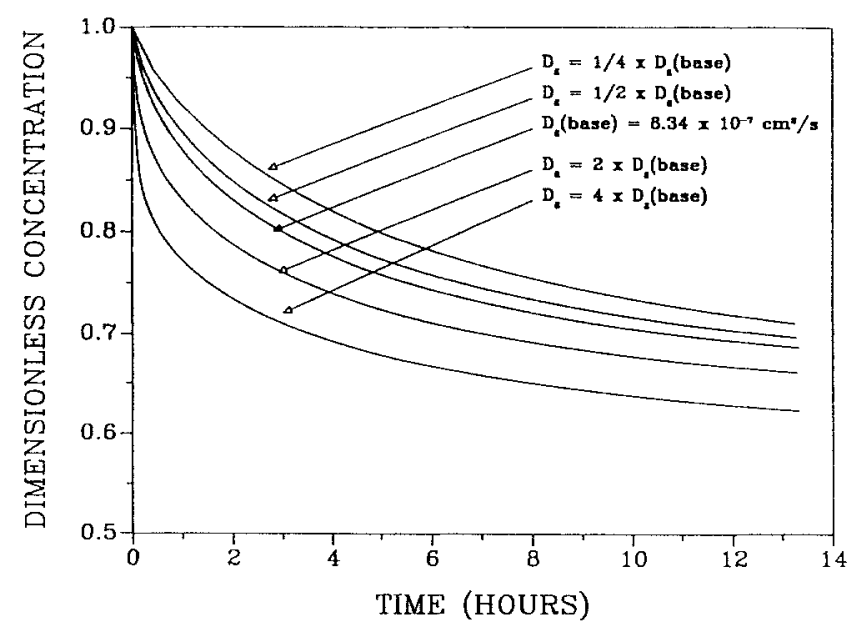

Figure 5. Sensitivity of the bulk concentration of $\beta$-lactamse on the diffusivity within the membrane. Diffusivity within the core held constant at $D_{c}=6.0 \times 10^{-7} \mathrm{~cm}^{2} / \mathrm{s}$.

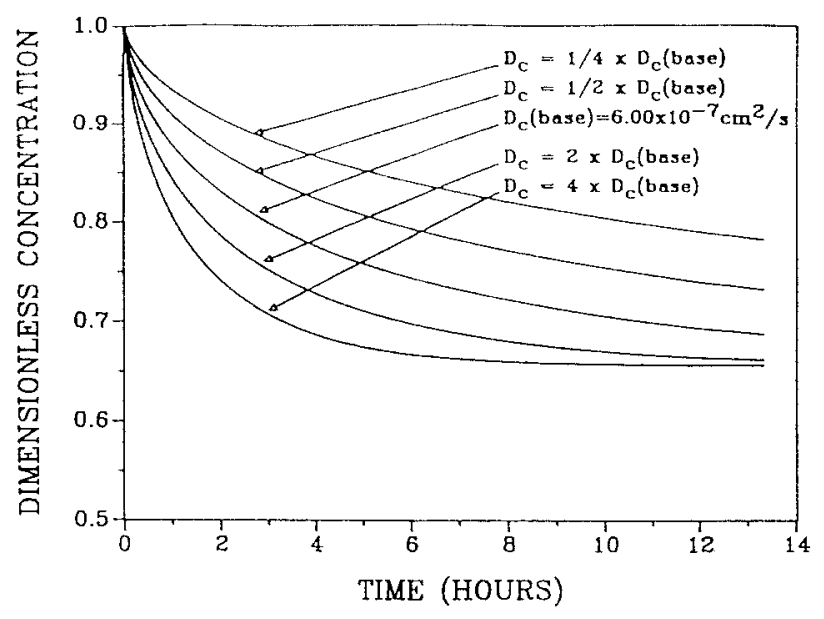

Figure 6. Sensitivity of the bulk concentration of $\beta$-lactamase on the diffusivity within the core.

Diffusivity within the membrane held constant at $D_{g}=8.34 \times 10^{-7}$ $\mathrm{cm}^{2} / \mathrm{s}$.

alginate gel beads containing no adsorbent using the methodology of Tanaka (1984). Because of the formulation of the material balance equations, the diffusivities appearing in Table 4 have been divided by the corresponding porosities.

It was found that the effective diffusivities of $\beta$-lactamase and albumin in the alginate membrane are nearly $1 / 2$ and $1 / 4$ of their expected free solution diffusivities (Setlow and Pollard, 1962; Cantor and Schimmel, 1980).

\section{Simulation Studies for Various Design Parameters of Gel-Entrapped Adsorbents}

Unless otherwise stated, all simulations are carried out under excess albumin concentration.

\section{Effect of bead size}

Equations 2 to 8 indicate that the profile of dimensionless concentration with respect to dimensionless time is independent

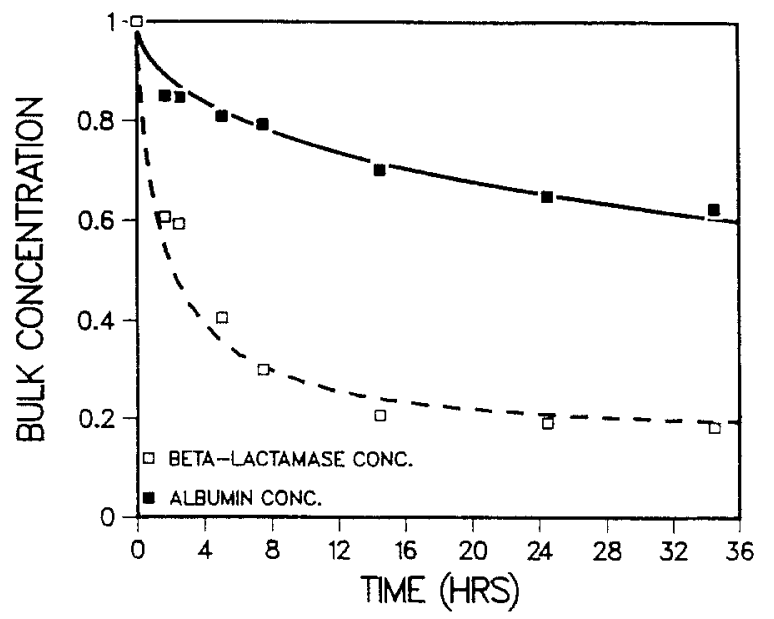

Figure 7. Comparison of experimental and simulated bulk concentration profiles during the competitive batch adsorption of $\beta$-lactamase and albumin using alginate-encapsulated DEAE-trisacryl. 


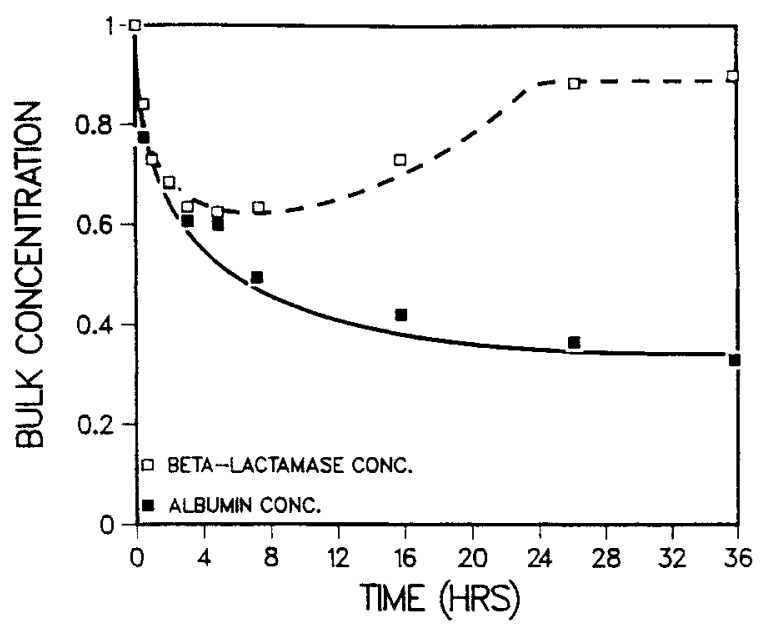

Figure 8. Comparison of experimental and simulated bulk concentration profiles during the competitive batch adsorption of $\beta$-lactamase and albumin using agarose-entrapped DEAE-trisacryl.

of the bead size as long as the same ratio of the crude solution to the adsorbent (i.e., same $\alpha$ ) is utilized during the batch adsorption. The radius of the bead, however, influences the time scale of the adsorption process since normalizing time constant, $t^{*}$ directly varies with the square of the bead radius. Thus, in principle, the time of adsorption using gel-entrapped adsorbents can be reduced without sacrificing the kinetic purification or adsorption capacity by reducing the size of the beads. If kinetic separation effects are being exploited, the adsorption kinetics should be sufficiently delayed to allow enough time for the easy removal of adsorbent beads from the crude solution. The free adsorbent particles generally equilibrate very rapidly (in the order of a few minutes) with the crude solution allowing minimal time for the practical utilization of kinetic separation effects.

\section{Effect of the crude to bead volume ratio $(\alpha)$}

One way to enhance the adsorption kinetics is to increase the amount of adsorbent used per unit volume of the crude solution being processed. Figure 9 shows the simulation curves indicating the effect of changing $\alpha$ on the adsorption kinetics in the neighborhood of the experimental base case (case b). The simulation runs indicate that by using a smaller value of $\alpha$ it is possible to enhance the kinetic adsorption of the smaller bioproduct ( $\beta$-lactamase). Figure 9 shows that when the value of $\alpha$ was reduced from 10.44 by two- and three-folds, the corresponding increase in the amount of $\beta$-lactamase adsorbed was approximately 1.9 and 2.8 folds, respectively. The corresponding increase in the adsorption of albumin for the same cases was

Table 4. Diffusion Coefficients of $\beta$-Lactamase and Albumin in Different Regions of Immobilized Adsorbents

\begin{tabular}{lccc}
\hline & $\begin{array}{c}\text { Alginate } \\
\text { Membrane } \\
\mathrm{cm}^{2} / \mathrm{s}\end{array}$ & $\begin{array}{c}\text { Alginate } \\
\text { Capsule Core } \\
\mathrm{cm}^{2} / \mathrm{s}\end{array}$ & $\begin{array}{c}\text { Agarose-Entrapped } \\
\text { Adsorbent Beads } \\
\mathrm{cm}^{2} / \mathrm{s}\end{array}$ \\
\hline$\beta$-Lactamase & $8.34 \times 10^{-7}$ & $6.00 \times 10^{-7}$ & $2.80 \times 10^{-7}$ \\
Albumin & $5.36 \times 10^{-7}$ & $2.2 \times 10^{-8}$ & $1.55 \times 10^{-8}$ \\
\hline
\end{tabular}

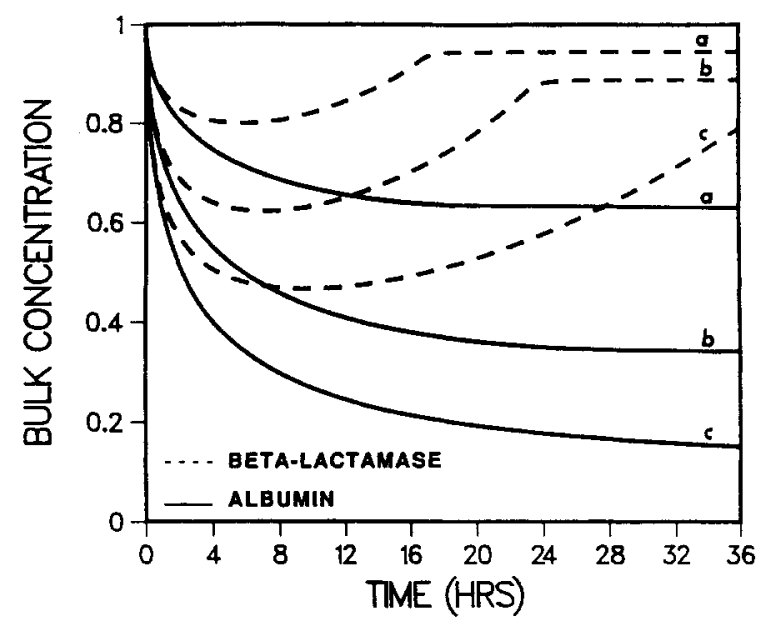

Figure 9. Simulated bulk concentration profiles for the adsorption of $\beta$-lactamase and albumin using agarose-entrapped DEAE-trisacryl showing the effect of crude to bead volume ratio $(\alpha)$.

(a) $\alpha=10.44$; (b) $\alpha=10.44 / 2=5.22$; (c) $\alpha=10.44 / 3=3.48$

approximately 1.8 and 2.3 folds. The reason for the lower increase in the adsorption of albumin is because its concentration in the bulk solution decreases close to exhaustion at the low values of $\alpha$.

\section{Effect of the adsorbent content}

Another approach to increase the amount of adsorbent used per unit volume of the crude solution is to increase the binding capacity of the beads by increasing their adsorbent content. Figure 10 shows the effect of varying the adsorbent content of the gel-entrapped beads in the neighborhood of the experimental base case (case b). It was found that as the adsorbent content is increased both the overall adsorption and the adsorption kinetics are enhanced for the product ( $\beta$-lactamase) and the contami-

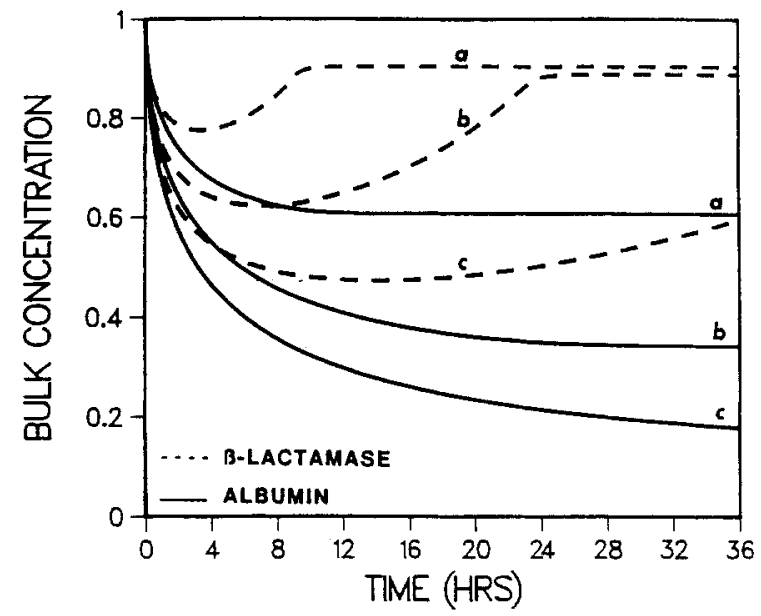

Figure 10. Simulated bulk concentration profiles for the adsorption of $\beta$-lactamase and albumin using agarose-entrapped DEAE-trisacryl showing the effect of adsorbent content in the bead $\left(\rho_{b E}\right)$.

(a) $\rho_{b}=0.317 \mathrm{~g}$ adsorbent $/ \mathrm{mL}$; (b) $\rho_{b}=0.317 \times 2=0.634 \mathrm{~g}$ adsorbent $/ \mathrm{mL}$; (c) $\rho_{b}=0.317 \times 3=0.951 \mathrm{~g}$ adsorbent $/ \mathrm{mL}$ 
nant (albumin). In terms of magnitude, however, the amount of increase was slightly poorer compared to the earlier described strategy of changing $\alpha$ (crude volume to bead volume ratio). As adsorbent content $\left(\rho_{b}\right)$ is increased by two- and three-folds, the corresponding increases in the adsorption of $\beta$-lactamase were 1.7 and 2.3 folds, respectively. The adsorption of albumin under same conditions increased by 1.7 and 2.1 folds, respectively. The upper limit of the adsorbent content of the beads is determined by the constraints posed by the mechanical strength of the final bead preparation and operational difficulties in manufacturing.

\section{Simulation Studies for Various Design Parameters of Gel-Membrane Encapsulated Adsorbents}

\section{Effect of capsule core size}

The material balance equations for membrane encapsulated adsorbents indicate that the relationship between dimensionless bulk concentration and dimensionless time depends on the ratio of the capsule radius to capsule-core radius as long as the ratio of crude volume to total capsule volume (i.e., $\alpha$ ) remains the same. Thus, similar to the case of gel-entrapped beads the time of adsorption using gel-encapsulated adsorbents can be reduced without sacrificing the kinetic purification or adsorption capacity by reducing the size of the capsules while maintaining the same ratio of the capsule radius to core radius.

\section{Role of capsule membrane thickness}

An extra design factor is the thickness of the capsule membrane. The primary factor influencing the choice of membrane thickness is the mechanical strength of the capsule preparation. In our experimental preparations, alginate membranes ranging in thickness from $100-200 \mu \mathrm{m}$ were found to be sufficient to assure good mechanical strength. This factor, however, may vary in different applications requiring different agitation and shear conditions. To investigate the effect of membrane transport in our capsule preparations, simulations were carried out to obtain dynamic product and contaminant concentration profiles for the base case, i.e., capsules having no enclosing membrane. It is impossible to conduct such a physical experiment since an enclosing membrane is required to maintain the integrity of the capsules. Simulation of this hypothetical capsule with an infinitely thin membrane can allow us to evaluate the contribution of diffusional resistances within the membrane in determining the bulk concentration profiles. Figure 11 shows the comparison of two sets of concentration profiles. Though the added membrane thickness marginally reduces the adsorption rate of the product, there is very little influence on the overall concentration profile. This result can be explained partly from the significantly lower diffusion coefficient values of both $\beta$-lactamase and albumin in the capsule core compared to those in the alginate gel-membrane (see Table 4).

\section{Influence of the adsorbent content within the capsule core}

In the earlier discussion on the role of adsorbent content in the case of gel-entrapped beads, it was found that high adsorbent content can give rise to higher kinetic binding capacity. In the experimental case involving the adsorption of $\beta$-lactamase and albumin using alginate-encapsulated DEAE-Trisacryl, the adsorbent particles are present in a tightly packed form. In fact, the adsorbent content of the capsule core $(1.27 \mathrm{~g}$ adsorbent $/ \mathrm{mL}$

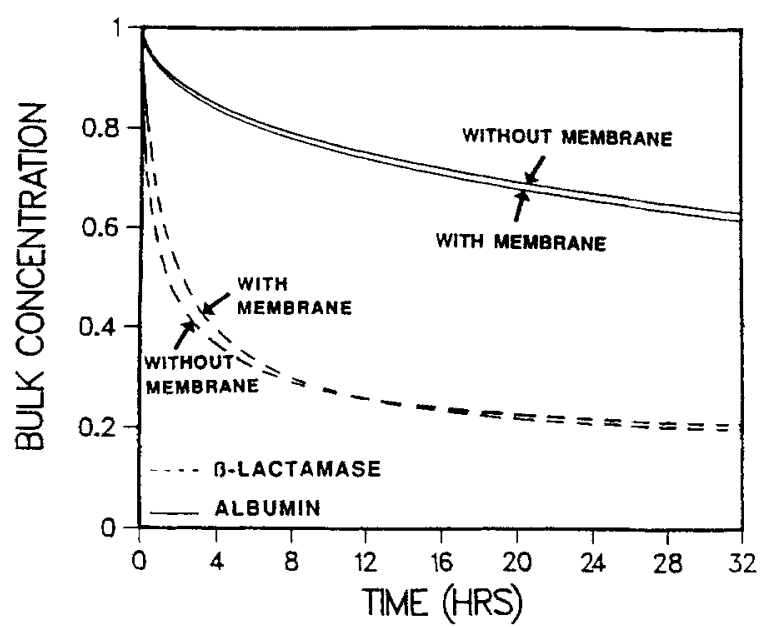

Figure 11. Simulated bulk concentration profiles for the adsorption of $\beta$-lactamase and albumin using alginate-encapsulated adsorbents showing the effect of membrane thickness.

capsule core) was found to be higher than what was observed in a gravity packed column (approximately $1.0 \mathrm{~g}$ adsorbent $/ \mathrm{mL}$ ). The effective diffusion coefficient of albumin was restricted by a factor of $1 / 100$ while that of $\beta$-lactamase was restricted only by a factor of $1 / 10$ compared to free solution diffusivities. $A$ high ratio of the effective diffusivities of $\beta$-lactamase and albumin $(\sim 30)$ in the capsule core probably accounts for the significantly improved kinetic separation observed in the case of alginate encapsulated DEAE-Trisacryl compared to agarose entrapped DEAE-Trisacryl beads.

\section{Effect of crude to capsule volume ratio ( $\alpha$ )}

As discussed in the previous section, the adsorbent content of the capsule core cannot be readily increased, if it is already present in a tightly packed or compressed form. The only other way to increase the ratio of adsorbent used per unit volume of crude processed is to use more capsules per unit batch volume, thereby decreasing $\alpha$. Figure 12 shows the simulation curves indicating the effect of changing $\alpha$ on the adsorption kinetics. Simulation curves in Figure 12 indicate that by using a smaller value of $\alpha$ it is possible to enhance the kinetic adsorption of both $\beta$-lactamase and albumin. A two- and three-fold increase in the number of capsules per unit volume at the end of a 36-hour adsorption period increased $\beta$-lactamase binding only by 1.25 and 1.34 fold, respectively. Albumin bound at the end of same period increased by 1.81 and 2.45 folds, respectively.

\section{Discussion of Results}

A mathematical model was developed to describe multicomponent diffusion and binding in both gel-entrapped and membraneencapsulated types of gel-enclosed adsorbent preparations. The model was successfully employed to describe both single and two-component adsorption behavior on gel-entrapped and membrane-encapsulated DEAE-trisacryl adsorbents using solutions containing purified $\beta$-lactamase, purified human serum albumin, and their mixtures. It is evident that the agreement between the theory and the experimental data for batch adsorption using gel-enclosed adsorbents in both entrapped and encapsulated forms is generally good. The model in its current form 


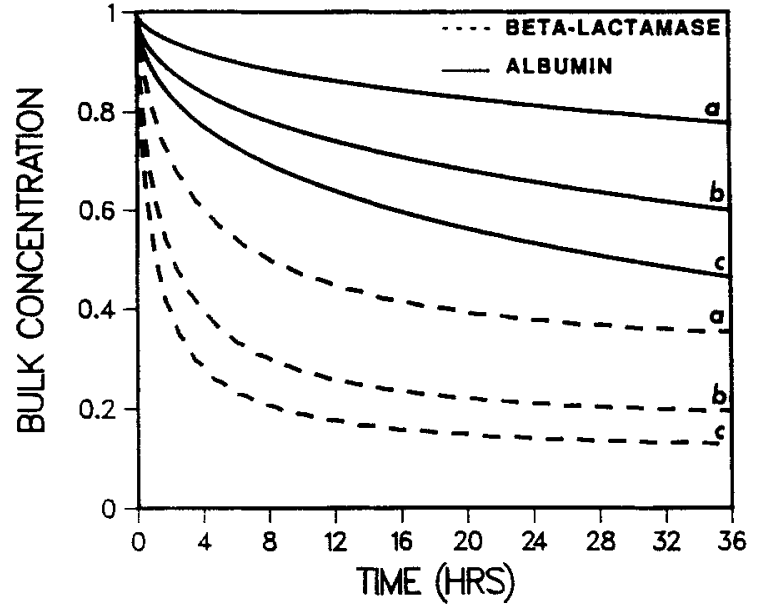

Figure 12. Simulated bulk concentration profiles for adsorption of $\beta$-lactamase and albumin using alginate-encapsulated adsorbents showing the effect of crude to capsule core volume ratio $\left(\alpha^{\prime}\right)$.

(a) $\alpha^{\prime}=10.44$; (b) $\alpha^{\prime}=10.44 / 2=5.22$; (c) $\alpha^{\prime}=10.44 / 3=$ 3.48

has the capability to predict the trends of multicomponent adsorption with changes in different design parameters. The model allowed successful prediction of the typical $U$-shaped dynamic adsorption curves obtained for $\beta$-lactamase in $\beta$-lactamase/albumin model system. Several parameters were needed to obtain bulk concentration profiles using this model. These parameters included the multicomponent equilibrium adsorption isotherms, effective diffusivities in different regions and the geometric parameters, and various porosities. Effective diffusivity within the agarose-entrapped adsorbent bead and the core of alginate capsules were utilized as an adjustable parameter.

The values of effective $\beta$-lactamase and albumin diffusivities required to fit the experimental batch adsorption data gave an idea of the key diffusional resistances contributing to the kinetic separation process. The diffusion of albumin in the core of alginate capsules was significantly retarded compared to that of $\beta$-lactamase. The significant retardation of albumin diffusion was the key factor behind the superior performance of alginateencapsulated DEAE-Trisacryl in adsorbing $\beta$-lactamase. This greater reduction in the diffusivity of larger macromolecules was expected from the models for pore diffusion developed using hydrodynamic theory (Paine and Scherr, 1975). We speculate that this greater reduction in diffusivities within the core is due to membrane shrinkage during the curing process which in turn compresses the adsorbent particles, increasing the adsorbent density within the core up to $1.27 \mathrm{~g} / \mathrm{mL}$ (Table 3). At such a density, the diffusivity in the core primarily reflects diffusion through the porous adsorbent particles. The reduction in the diffusion coefficients within the core is similar to values obtained for other proteins of the same size range in similar porous matrices (Graham and Fook, 1982; Colton et al., 1979; Paine and Scherr, 1975). Comparatively, the adsorbent density in the agarose-entrapped adsorbents was $(0.634 \mathrm{~g} / \mathrm{mL})$.

Simulation studies also revealed that the diffusional resistance provided by alginate membrane did not contribute significantly to the two-component bulk concentration profiles when alginate-encapsulated DEAE-trisacryl was employed. It was found that the two proteins experience significantly more different porosities in the gel membrane than in the capsule core as shown in Table 3. It is difficult to postulate exactly why two proteins experience similar porosity in the capsule core but somewhat different values in the gel membrane. In the absence of a detailed study, we can speculate that the higher porosity for $\beta$-lactamase in the gel membrane is probably due to its smaller size compared to albumin. This thinking is consistent with the results of Tanaka et al. (1984) who found significant differences in the accessibility of albumin and lactalbumin in alginate matrices. It was found that both $(\alpha)$ and adsorbent content within the capsules or beads could be effectively utilized to enhance both the adsorption rate and kinetic binding of the desired bioproduct. These factors, however, have physical limits governed by processing and manufacturing constraints. Using either of the above strategies, the increase in the binding capacity or the adsorption rate was not always proportional to the amount of extra adsorbent employed. An evaluation of process economics may be the only way to optimize the amount of adsorbent used to process a given quantity of crude solution.

Dimensional analysis of governing equations revealed further information on the influence of geometric design parameters such as the bead/capsule size and membrane thickness. In the dimensionless form, the bulk concentration profiles were found to be independent of the size of the gel-enclosed adsorbent preparation as long as $(\alpha)$ and the ratio of capsule to core radius remained the same. Under these conditions, the dimensionless bulk concentration profiles show identical separation characteristics. Thus, adsorption time can be scaled down by using a smaller size of the adsorbent preparation. Another physical constraint is the need to have very uniform (monodisperse) beads or capsules to obtain maximum kinetic separation. A batch containing immobilized adsorbent beads having a wide size distribution would show poorer kinetic adsorption property because smaller capsules or beads would equilibrate faster than their larger counterparts.

\section{Acknowledgement}

The study was performed under Grant No. CBT-8812996, National Science Foundation.

\section{Notation}

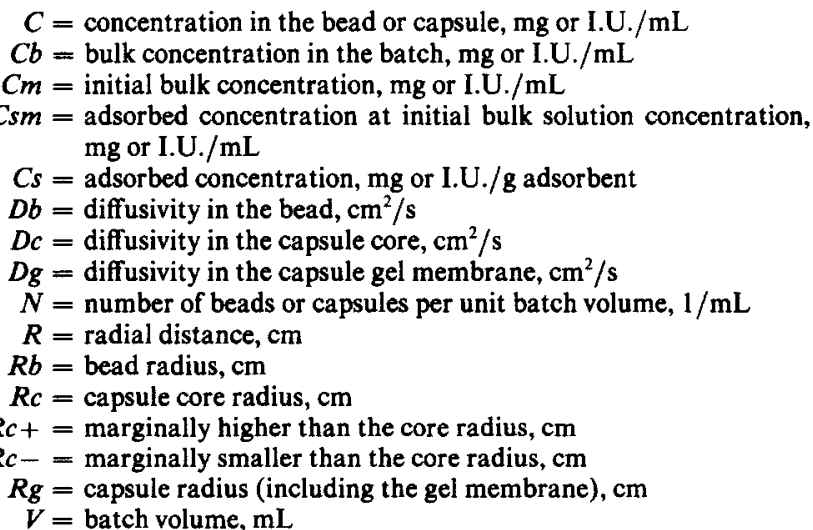

\section{Greek letters}

$$
\begin{aligned}
& \epsilon b=\text { bead porosity } \\
& \epsilon c=\text { capsule core porosity }
\end{aligned}
$$


$\epsilon g=$ gel membrane porosity

$\rho_{b}=$ adsorbent content in the bead, $\mathrm{g}$ adsorbent $/ \mathrm{ml}$ bead volume

$\rho_{c}=$ adsorbent content in the capsule core, $\mathrm{g}$ adsorbent $/ \mathrm{ml}$ capsule core volume

\section{Subscripts}

$i=i$ th component, $i$ varies from 1 to $n$

$n=$ total number of competing components

\section{Literature Cited}

Bradford, M., "A Rapid and Sensitive Method for the Quantitation of Microgram Quantities of Protein Utilizing the Principle of ProteinDye Binding," Anal. Biochem., 72, 248 (1976).

Cantor, C. R., and P. R. Schimmel, "Size and Shape of Macromolecules," Biophysical Chemistry, Part II, Chap. 10, 539, W. H. Freeman and Co., San Francisco (1980).

Carnahan, B., H. A. Luther, and J. O. Wilkes, Applied Numerical Methods, Wiley, New York (1969).

Chang, H. T., "Mathematical Modeling and Scanning Electron Microscopic Study of Biofilm on Adsorptive Media," PhD Thesis, University of Illinois, Urbana-Champaign (1985).

Chase, H. A., "Affinity Separations Using Monoclonal Antibodies-A New Tool for Biochemical Engineer," Chem. Eng. Sci., 39, 1009 (1984).

Colton, C. K., C. N. Satterfield, and C-J. Lai, "Diffusion and Partitioning of Macromolecules within Finely Porous Glass," AIChE J., 21, 289 (1975).
Graham, E. E., and C. F. Fook, "Rate of Protein Adsorption and Desorption on Cellulosic Ion Exchangers," AIChE J., 28, 245 (1982).

Horstmann, B. J., and H. A. Chase, "Modeling the Affinity Adsorption of Immunoglobulin $\mathbf{G}$ to Protein A Immobilized to Agarose Matrices," Chem. Eng. Res. Des., 67, 243 (1989).

Nguyen, A.-L., and J. H. T. Luong, "Diffusion in Karragenen Gel Beads," Biotech. Bioeng., 28, 1261 (1986).

Nigam, S. C., A. Sakoda, and H. Y. Wang, "Bioproduct Recovery from Unclarified Broths and Homogenates Using Immobilized Adsorbents," Biotechnol. Prog., 4, 166 (1988).

Nigam, S. C., "Bioproduct Recovery from Whole Broths Using Immobilized Adsorbents," PhD Thesis, University of Michigan, Ann Arbor (1988).

Paine, P. L., and P. Scherr, "Drag Coefficients for the Movement of Rigid Spheres through Liquid Filled Cylindrical Pores," Biophys. J., 15, 1087 (1975).

Setlow, R. B., and E. C. Pollard, "Physical Methods for Determining the Sizes and Shapes of Molecules," Molecular Biophysics, Chap. 4, 77, Addison-Wesley Publishing, Reading, MA (1962).

Tanaka, H., M. Matsumura, and I. A. Veliky, "Diffusion Characteristics of Substrates in Calcium Alginate Gel Beads," Biotech. Bioeng., 26, 53 (1984).

Tsou, H-S, and E. E. Graham, "Prediction of Adsorption and Desorption of Protein on Dextran Based Ion-Exchange Resins," AIChE J., 31, 1959 (1985).

Wang, H. Y., and K. Sobnosky, "Design of a New Affinity Adsorbent for Biochemical Product Recovery," ACS Symp. Ser., 271, 123 (1984).

Manuscript received Jan. 23, 1989, and revision received June 19, 1990. 